\title{
Features of DLW-STED nanolithography for quantum optics
}

\author{
A.G. Vitukhnovsky ${ }^{1,2,{ }^{*}, \text { D.A. Chubich }}{ }^{2}$, D.A. Kolymagin ${ }^{2}$, and R.D. Zvagelsky ${ }^{1,2}$ \\ ${ }^{1}$ Lebedev Physical Institute RAS, 119991 Moscow, Russia \\ ${ }^{2}$ Moscow Institute of Physics and Technology 141701 Dolgoprudny, Moscow Region, Russia
}

\begin{abstract}
The report proposes a discussion of the effective combination of two current trends: the additive 3D printing method and the development of multicomponent photonic schemes, as well as the development of the foundations of additive scalable and flexible optical technology for creating interconnects and optical structures, which can solve problems such as the creation of interchip optical compounds, various optical and quantum-optical systems on a chip (resonators, modulators, photon detectors, single-photon radiation sources, etc.).
\end{abstract}

Presentation discusses the process of combining two relevant areas: the additive 3D printing method and the development of multicomponent photonic integrated circuits (PIC). Varieties of photonic systems are already actively used in various applications, but their designs are two-dimensional due to the limitations of lithographic techniques.

Hence, great restrictions on the potential capabilities of photonic systems as a whole follow. To overcome these shortcomings, it is necessary to develop the foundations of an additive scalable and flexible optical technology for creating interconnects and optical structures, which will be able to solve problems such as the creation of interchip optical compounds, a variety of optical and quantum-optical systems on a chip (resonators, modulators, photon detectors, single-photon radiation sources, etc.) [1].

A new technology of optical connections (connectors, PWBs - Polymer Wire Bonds, Fig. 1.) between the elements of photonic integrated circuits (PIC) by two-photon femtosecond photolithography using both direct laser writing (Direct Laser Writing-DLW) and using an additional quenching laser (STimulated Emission Depletion-STED) [2].

To solve the problems of quantum optics, we need single-photon emitters with quantum dots, reaching a maximum average number of photons per pulse $\langle n\rangle=1$. We need a fast source in the $\mathrm{GHz}$ range at the telecommunication wavelength (where optical fiber losses are minimal), and a narrow emission line width to ensure multiplexing with separation by wavelength and, therefore, the possibility of further increasing the speed of quantum key distribution $(\mathrm{QKD})$. Colloidal quantum dots are especially interesting for this purpose due to recent improvements in their purity and stability of single-photon radiation.

\footnotetext{
*Corresponding author: vitukhnovsky@ mail.ru
} 


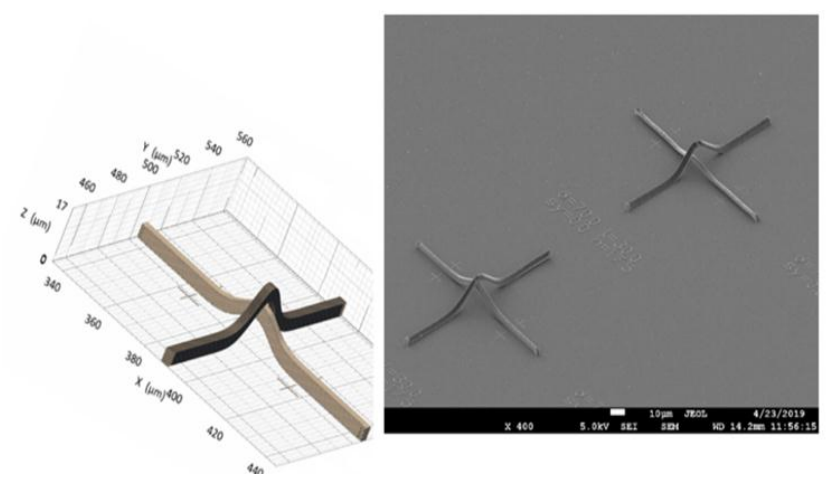

Fig. 1. SEM image of PWB (photon connectors) and PWB model

Using semi-contact atomic force microscopy, we studied the dimensional parameters of planar and polymer 3D waveguides (photon wires - PWB) and, first of all, their height from the surface of silicon nitride and the root mean square roughness $(15 \mathrm{~nm})$ were determined. Hybrid three-dimensional optical connectors allow you to successfully turn on light from efficient sources of single photons based on colloidal quantum dots and nitrogen and silicon substituted vacancies (NV and Si-centers) in diamond, incorporated into the created polymer PWB, into planar waveguides for subsequent use in photonic integrated schemes.

The creation of hybrid systems combining standard planar chips and polymer 3D connectors ("bridges") is promising and allows you to significantly expand the optical functionality that cannot be obtained in 2D geometry for PIC. [3]

The technological part of the work was supported by the Russian Science Foundation grant No. 1519-00205, and the research was supported by the Russian Foundation for Basic Research grants 1802-00811, 16-29-11805 and 18-29-20129.

\section{References}

1. L. Elsinger, R. Gourgues, I. Zaden et al, Nano Lett., 19, 8 (2019)

2. A.G. Vitukhnovsky, D.A. Glubokov, V.V. Sychev, I.V.Taidakov, RF patent for invention No. 2510632 (priority November 20, 2012)

3. D.A. Chubich, R.D. Zvagelsky, A.G. Vitukhnovsky et al, J. Phys. D (to be published) 